\title{
The traditional knowledge on edible wild leafy vegetables of Rabha Tribe in Duars of North Bengal: a potential reinforcement to food security
}

\author{
Ajita Sarkar ${ }^{1}$ and Abhaya Prasad Das ${ }^{2}$ \\ ${ }^{1}$ Corresponding author: Fagu, Gorubathan-735 231, Kalimpong, West Bengal, India \\ ajitasarkar2007@rediffmail.com \\ ${ }^{2}$ Department of Botany, Rajiv Gandhi University, Rono Hills, Doimukh 791 112, Arunachal Pradesh, India \\ [Received 21.11.2018; Revised 24.12.2018; Accepted 26.12.2018; Published 31.12.2018]
}

\begin{abstract}
s
Northern part of West Bengal is home to diverse group of tribal people coming from different roots of origin. These people have rich tradition and cultural backgrounds. Among them Rabha is one of the primitive tribes. This paper primarily deals with the recording of their traditional knowledge about the edible leafy vegetables and analyzing various preparations. As per the latest Nagoya Protocol, open ended questionnaires were used to obtain information from the resource persons with prior written permissions. In total 100 informants were interviewed that yielded the names of 40 vegetable plants belonging to 26 families. Among all Amaranthaceae recorded the maximum of eight species, followed by Rubiaceae with four speciess. For rest of the family either two or at least one species could be recorded. In almost all cases, tender shoots or leaves were cooked and consumed, whereas only in case of vegetable plants like Alternanthera paronichioides, Oldenlandia corymbosa, Polycarpon prostratum and Portulaca oleracea were reported to be cooked wholly. Some of these species also has reported medicinal values and are often eaten as part of traditional system of treatment for different ailments. The database of such plants can help in solving problems of food securities by utilizing traditional knowledge of Rabha people or in larger perspective any other tribes.
\end{abstract}

Key words: Rabha, Wild leafy vegetables, Tribes, West Bengal

\section{INTRODUCTION}

North Bengal, the northernmost part of West Bengal, is known for its vast resources of flora and fauna. This part of West Bengal is surrounded by Indian states like Assam on East, Sikkim on North and Bihar on West and at the same time shares international borders with Nepal on West, Bhutan on North and Bangladesh on Southern region. Northern part of West Bengal includes nine districts namely Malda, Murshidabad, Dakshin Dinajpur, Uttar Dinajpur, Darjeeling, Kalimpong, Jalpaiguri Coochbehar and Alipurduar, spread between coordinates of $25.01^{\circ} \mathrm{N}$ and $88.14^{\circ} \mathrm{E}$ to $26.6476^{\circ} \mathrm{N}$ and $89.4135^{\circ} \mathrm{E}$ (Egiye Bangla 2018). For past thousands of years, these vast lands have been home to diverse group of ethnic peopleand aboriginal tribes who lived here with their rich tradition and cultural backgrounds (Roy 2016). Despite rapid percolation of modern culture and amenities, most of these tribes have managed to conserve their precious traditional knowledge by practicing it in their day to day life. Rabha is one such primitive tribes living in the plains. Majority of them are spread over in western Assam, Meghalaya and North-eastern part of West Bengal (mainly Alipurduar, Jalpaiguri and Cooch Behar Districts) (Roy 2016). Rabhas are basically farmers and earn their livelihood through agriculture and by producing and selling their handicrafts. Their population is higher in villages with large agricultural lands with forests at vicinity (Basumatary 
2010). As per Das and Teron (2014), Rabhas have supposedly developed a valuable ethnobotanical knowledge base which has helped them in sustainable utilization of plant resources around them. This has helped their population in sustainably surviving for many decades in scarce agricultural resources.

Considering present situation of food scarcity in the world, traditional knowledge of this kind can be an important aid to establish better food security (Yongabi \& DeLuca 2015). Using and exploiting this knowledge base of wild edible leafy vegetables of Rabha tribe can be boon in reducing hunger. As the Indian population has already crossed the 1.3 billion mark, limited agricultural system finds it difficult to produce more food for this rapidly growing mass. Focusing on using non-exploited resources judiciously should be the right approach. Since, most of the people consume already grown food or grow food themselves, however, in developing countries like India, the buying capacity of majority of people is very weak (WESP 2018). Therefore, government should press upon growing foods that require least maintenance, low or no chemical inputs and more resilient to climate change (FAOUN 2018). Knowing about such food can come from the knowledge base of indigenous people. This paper by and large focuses on the traditional knowledge base of Rabha tribe of North Bengal about the wild edible leafy vegetables and their various preparations. Having knowledge of these largely unknown resources can add to dealing with food scarcity among mainstream populace.

\section{MATERIALS AND METHODS}

\section{Study Area}

This paper primarily focused on Rabha tribe and their knowledge of edible leafy vegetables. In West Bengal, Rabhas are primarily concentrated in the North-eastern part, generally referred as Duars and politically represented by three districts, namely Alipurduar, Jalpaiguri, and Cooch Behar, and was selected for the data collection. The survey locations covered an estimated area of $9814 \mathrm{~km}^{2}$ spread across $25^{\circ} 57^{\prime} \mathrm{N}$ to $26.59^{\circ} \mathrm{N}$ Latitude and 88p $232 \mathrm{E}$ to $89^{\circ} 53^{\prime}$ E Longitude (Egiye Bangla 2018). These districts are bound by Assam at East, Bhutan at North, Bangladesh Soudeth and Darjeeling and Kalimpong districts at South-western region. The average rainfall is $3500 \mathrm{~mm}$, with mean relative humidity 86 percent (Egiye Bangla 2018).The climatic character is sub-tropical and humid with an average temperature ranging between $6^{\circ} \mathrm{C}$ and $33^{\circ} \mathrm{C}$ (winter and summer). Whole region is richly endowed with seasonal as well continual rivers and rivulets originating from Sikkim, Bhutan and Darjeeling hills. The major rivers these districts are Teesta, Jaldhaka, Murti, Torsha, Raidak, Kaljani, Sankosh, etc (Egiye Bangla 2018).

\section{Data Collection}

Open-ended questionnaire developed based on the initial understandings with the local respondents, was used to collect the information regarding the leafy vegetable plants and their preparations. Village heads, resource persons and locals were elaborately interviewed for more information. Since this work was primarily based on the knowledge-base of local Rabha tribe therefore the data was collected as per the guidelines of Nagoya protocol (2011) by Participatory Rural Appraisal (PRA) method in order to ensure active involvement of the locals (Mukherjee 1993; Campbell 2001). Prior published works on edible plants and Rabhas of this region were substantiated as secondary information (Roy 2016; Das \& Teron 2014; Chowdhury 2012; Narzary et al. 2016; Adhikary \& Barman 2014) which were corroborated with locals for validation. Specimen of leafy vegetables were collected, identified and deposited at NBU Herbarium, Department of Botany, University of North Bengal. 


\section{RESULTS AND DISCUSSION}

In total 100 informants were interviewed which yielded the names of 40 vegetable plants belonging to 26 families (Table 1). Among all, Amaranthaceae recorded the maximum species with eight numbers, followed by Rubiaceae with four numbers. For rest of the family either two or at least one species could be recorded. In almost all cases, tender shoots or leaves were cooked and consumed, whereas only in case of vegetable plants like Alternanthera paronichioides, Oldenlandia corymbosa, Polycarpon prostratum and Portulaca oleracea were reported to be cooked wholly. Some of these species also has reported medicinal values and are often eaten as part of traditional system of treatment for different ailments. The database of such plants can help in solving problems of food securities by utilizing traditional knowledge of Rabha people or in larger perspective any other tribes.

Table 1. Wild Leafy Vegetables of Rabhas in Duars of West Bengal

\begin{tabular}{|c|c|c|c|c|}
\hline $\begin{array}{l}\text { Botanical Name [Family]; specimen } \\
\text { cited }\end{array}$ & $\begin{array}{l}\text { Rabha } \\
\text { name }\end{array}$ & Habit & $\begin{array}{l}\text { Edible parts and } \\
\text { consumption } \\
\text { pattern }\end{array}$ & $\begin{array}{l}\text { Additional } \\
\text { uses }\end{array}$ \\
\hline $\begin{array}{l}\text { Acmella calva (DC.) R.K.Jansen } \\
\text { [Compositae]; Ajita \& AP Das } 118\end{array}$ & Samnami & Herb & $\begin{array}{l}\text { Young leaves } \\
\text { cooked as } \\
\text { vegetable }\end{array}$ & Ethnomedicine \\
\hline $\begin{array}{l}\text { Alternanthera paronychioides A. St. } \\
\text { Hill [Amaranthaceae]; Ajita \& AP Das } \\
276\end{array}$ & - & Herb & $\begin{array}{l}\text { Whole plants } \\
\text { cooked as } \\
\text { vegetable }\end{array}$ & \\
\hline $\begin{array}{l}\text { Alternanthera philoxeroides (Mart.) } \\
\text { Griseb. [Amaranthaceae]; Ajita \& AP } \\
\text { Das } 243\end{array}$ & - & Herb & $\begin{array}{l}\text { Leafy-twigs as } \\
\text { vegetable }\end{array}$ & \\
\hline $\begin{array}{l}\text { Alternanthera sessilis (L.) R.Br. ex } \\
\text { DC. [Amaranthaceae]; Ajita \& AP Das } \\
254\end{array}$ & $\begin{array}{l}\text { Resang- } \\
\text { mama }\end{array}$ & Herb & $\begin{array}{l}\text { Fried leafy-twigs } \\
\text { eaten }\end{array}$ & \\
\hline $\begin{array}{l}\text { Amaranthus blitum subsp. oleraceus } \\
\text { (L.) Costea [Amaranthaceae]; Ajita \& } \\
\text { AP Das } 274\end{array}$ & Kudum & Herb & $\begin{array}{l}\text { Young leafy- } \\
\text { twigs as vegetable }\end{array}$ & \\
\hline $\begin{array}{l}\text { Amaranthus spinosus L. } \\
\text { [Amaranthaceae]; Ajita \& AP Das } 103\end{array}$ & Kudumfisa & Herb & $\begin{array}{l}\text { Young shoots } \\
\text { cooked as } \\
\text { vegetables }\end{array}$ & \\
\hline $\begin{array}{l}\text { Amaranthus viridis } \mathrm{L} . \\
\text { [Amaranthaceae]; Ajita \& AP Das } 275\end{array}$ & Kudum & Herb & $\begin{array}{l}\text { Young shoots } \\
\text { cooked as } \\
\text { vegetables }\end{array}$ & \\
\hline $\begin{array}{l}\text { Boerhavia diffusa L. [Nyctaginaceae]; } \\
\text { Ajita \& AP Das } 218\end{array}$ & Samkanka & Herb & $\begin{array}{l}\text { Leaves fried and } \\
\text { consumed }\end{array}$ & Medicinal \\
\hline $\begin{array}{l}\text { Centella asiatica }(\mathrm{L} .) \text { Urb. } \\
\text { [Umbelliferae]; Ajita \& AP Das } 053\end{array}$ & $\begin{array}{l}\text { Mochatn- } \\
\text { achar }\end{array}$ & Herb & $\begin{array}{l}\text { Whole plants } \\
\text { cooked as } \\
\text { vegetables }\end{array}$ & Medicinal \\
\hline
\end{tabular}




\begin{tabular}{|c|c|c|c|c|}
\hline $\begin{array}{l}\text { Botanical Name [Family]; specimen } \\
\text { cited }\end{array}$ & $\begin{array}{l}\text { Rabha } \\
\text { name }\end{array}$ & Habit & $\begin{array}{l}\text { Edible parts and } \\
\text { consumption } \\
\text { pattern }\end{array}$ & $\begin{array}{l}\text { Additional } \\
\text { uses }\end{array}$ \\
\hline $\begin{array}{l}\text { Chenopodium album } \mathrm{L} . \\
\text { [Amaranthaceae]; Ajita \& AP Das } 298\end{array}$ & - & Herb & $\begin{array}{l}\text { Leaves cooked as } \\
\text { vegetable }\end{array}$ & \\
\hline $\begin{array}{l}\text { Coccinia grandis (L.) Voigt } \\
\text { [Cucurbitaceae]; Ajita \& AP Das } 123\end{array}$ & - & Climber & $\begin{array}{l}\text { Young leafy- } \\
\text { twigs cooked as } \\
\text { vegetable }\end{array}$ & $\begin{array}{l}\text { Medicinal, rice } \\
\text { beer } \\
\text { preparation }\end{array}$ \\
\hline $\begin{array}{l}\text { Colocasia esculenta (L.) Schott } \\
\text { [Araceae]; Ajita \& AP Das } 050\end{array}$ & $\begin{array}{l}\text { Lheng } \\
\text { Basar }\end{array}$ & Herb & $\begin{array}{l}\text { Whole young } \\
\text { plant cooked as } \\
\text { vegetables }\end{array}$ & \\
\hline $\begin{array}{l}\text { Commelina benghalensis L. } \\
\text { [Commelinaceae]; Ajita \& AP Das } 319\end{array}$ & Surung & Herb & Fried leaves eaten & Fodder \\
\hline $\begin{array}{l}\text { Deeringia amaranthoides (Lam.) } \\
\text { Merr. [Amaranthaceae]; Ajita \& AP } \\
\text { Das } 085\end{array}$ & Janger & Climber & $\begin{array}{l}\text { Young leafy } \\
\text { shoots cooked as } \\
\text { vegetables }\end{array}$ & Ethnomedicine \\
\hline $\begin{array}{l}\text { Diplazium esculentum (Retz.) Sw. } \\
\text { [Athyriaceae]; Ajita \& AP Das } 036\end{array}$ & - & Herb & $\begin{array}{l}\text { Tender fronds } \\
\text { cooked as } \\
\text { vegetable }\end{array}$ & \\
\hline $\begin{array}{l}\text { Drymaria cordata (L.) Willd. ex } \\
\text { Roem. \& Schult. [Caryophyllaceae]; } \\
\text { Ajita \& AP Das } 095\end{array}$ & - & Herb & $\begin{array}{l}\text { Fried whole } \\
\text { plants eaten }\end{array}$ & Ethnomedicine \\
\hline $\begin{array}{l}\text { Enydra fluctuans DC. [Compositae]; } \\
\text { Ajita \& AP Das } 441\end{array}$ & Jharaing & Herb & $\begin{array}{l}\text { Young leafy- } \\
\text { twigs cooked as } \\
\text { vegetable }\end{array}$ & Medicinal \\
\hline $\begin{array}{l}\text { Euphorbia hirta L. [Euphorbiaceae]; } \\
\text { Ajita \& AP Das } 311\end{array}$ & Dudeleka & Herb & $\begin{array}{l}\text { Leaves cooked as } \\
\text { vegetable }\end{array}$ & Medicinal \\
\hline $\begin{array}{l}\text { Glinus oppositifolius (L.) Aug.DC. } \\
\text { [Molluginaceae]; Ajita \& AP Das } 186\end{array}$ & - & Herb & $\begin{array}{l}\text { whole plants as } \\
\text { consumed }\end{array}$ & Medicinal \\
\hline $\begin{array}{l}\text { Helminthostachys zeylanica (L.) Hook. } \\
\text { [Ophioglossaceae]; Ajita \& AP Das } 216\end{array}$ & Musitop & Herb & $\begin{array}{l}\text { Tender leaves } \\
\text { cooked as } \\
\text { vegetables }\end{array}$ & \\
\hline $\begin{array}{l}\text { Houttuynia cordata Thunb. } \\
\text { [Saururaceae]; Ajita \& AP Das } 070\end{array}$ & Bagnetra & Herb & $\begin{array}{l}\text { Young leaves } \\
\text { pounded with } \\
\text { onion, green chilli } \\
\text { and salt taken as } \\
\text { pickle }\end{array}$ & \\
\hline $\begin{array}{l}\text { Ipomoea aquatica } \text { Forssk. } \\
\text { [Convolvulaceae]; Ajita \& AP Das } 079\end{array}$ & Kulum & Herb & $\begin{array}{l}\text { Young shoots } \\
\text { cooked as } \\
\text { vegetables }\end{array}$ & Medicinal \\
\hline
\end{tabular}


Ajita Sarkar \& A. P. Das 279

\begin{tabular}{|c|c|c|c|c|}
\hline $\begin{array}{l}\text { Botanical Name [Family]; specimen } \\
\text { cited }\end{array}$ & $\begin{array}{l}\text { Rabha } \\
\text { name }\end{array}$ & Habit & $\begin{array}{l}\text { Edible parts and } \\
\text { consumption } \\
\text { pattern }\end{array}$ & $\begin{array}{l}\text { Additional } \\
\text { uses }\end{array}$ \\
\hline $\begin{array}{l}\text { Leucas zeylanica (L.) W.T.Aiton } \\
\text { [Labiatae]; Ajita \& AP Das } 089\end{array}$ & Parbolang & Herb & $\begin{array}{l}\text { Young shoots } \\
\text { cooked as } \\
\text { vegetable }\end{array}$ & Medicinal \\
\hline $\begin{array}{l}\text { Lippia javanica (Burm.f.) Spreng. } \\
\text { [Verbenaceae]; Ajita \& AP Das } 111\end{array}$ & - & Shrub & $\begin{array}{l}\text { Young leaves } \\
\text { fried to eat; raw } \\
\text { leaves added to } \\
\text { curry as aromatic }\end{array}$ & \\
\hline $\begin{array}{l}\text { Marsilea minuta } \mathrm{L} \text {. [Marsileaceae]; } \\
\text { Ajita \& AP Das } 190\end{array}$ & Tengesi & Herb & $\begin{array}{l}\text { Fried leaves } \\
\text { consumed }\end{array}$ & Medicinal \\
\hline $\begin{array}{l}\text { Melia azedarach L. [Meliaceae]; Ajita } \\
\& \text { AP Das } 027\end{array}$ & $\begin{array}{l}\text { Bobaneemp } \\
\text { hang }\end{array}$ & Tree & $\begin{array}{l}\text { Fried leaves } \\
\text { consumed }\end{array}$ & \\
\hline $\begin{array}{l}\text { Momordica charantia L. } \\
\text { [Cucurbitaceae]; Ajita \& AP Das } 096\end{array}$ & - & Climber & $\begin{array}{l}\text { Leaves cooked as } \\
\text { vegetable }\end{array}$ & Medicinal \\
\hline $\begin{array}{l}\text { Moringa oleifera Lam. [Moringaceae]; } \\
\text { Ajita \& AP Das } 041\end{array}$ & Sojona & Tree & $\begin{array}{l}\text { Young fried } \\
\text { leaves consumed } \\
\text { as vegetables }\end{array}$ & Medicinal \\
\hline $\begin{array}{l}\text { Mussaenda glabra Vahl [Rubiaceae]; } \\
\text { Ajita \& AP Das } 193\end{array}$ & Kotmotia & Shrub & $\begin{array}{l}\text { Young fried } \\
\text { leaves eaten }\end{array}$ & \\
\hline $\begin{array}{l}\text { Mussaenda roxburghii Hook.f. } \\
\text { [Rubiaceae]; Ajita \& AP Das } 192\end{array}$ & Kotmotia & Shrub & $\begin{array}{l}\text { Young leaves } \\
\text { boiled in rice and } \\
\text { eaten }\end{array}$ & Ethnomedicine \\
\hline $\begin{array}{l}\text { Oldenlandia corymbosa } \mathrm{L} . \\
\text { [Rubiaceae]; Ajita \& AP Das } 233\end{array}$ & - & Herb & $\begin{array}{l}\text { Fried whole } \\
\text { plants eaten }\end{array}$ & Medicinal \\
\hline $\begin{array}{l}\text { Ophioglossum reticulatum } \mathrm{L} \text {. } \\
\text { [Ophioglossaceae]; Ajita \& AP Das } \\
300\end{array}$ & Suga & Herb & $\begin{array}{l}\text { Fried leaves } \\
\text { consumed }\end{array}$ & \\
\hline $\begin{array}{l}\text { Oroxylum indicum (L.) Kurz } \\
\text { [Bignoniaceae]; Ajita \& AP Das } 044\end{array}$ & Jamlao & Tree & $\begin{array}{l}\text { Tender shoots } \\
\text { soaked in hot } \\
\text { water and fried in } \\
\text { oil to consumed }\end{array}$ & Medicinal \\
\hline $\begin{array}{l}\text { Oxalis corniculata L. [Oxalidaceae]; } \\
\text { Ajita \& AP Das } 112\end{array}$ & Amainor & Herb & $\begin{array}{l}\text { Raw leaves } \\
\text { crushed with } \\
\text { green chilli and } \\
\text { salt to consume as } \\
\text { pickle }\end{array}$ & $\begin{array}{l}\text { Ethnomedicine, } \\
\text { plate cleaner }\end{array}$ \\
\hline $\begin{array}{l}\text { Paederia foetida L. [Rubiaceae]; Ajita } \\
\& \text { AP Das } 264\end{array}$ & Giphing & Climber & $\begin{array}{l}\text { Fried Young } \\
\text { leaves are eaten }\end{array}$ & Medicinal \\
\hline
\end{tabular}


Rabha tribe in North Bengal: wild edible vegetable

\begin{tabular}{|l|l|l|l|l|}
\hline $\begin{array}{l}\text { Botanical Name [Family]; specimen } \\
\text { cited }\end{array}$ & $\begin{array}{l}\text { Rabha } \\
\text { name }\end{array}$ & Habit & $\begin{array}{l}\text { Edible parts and } \\
\text { consumption } \\
\text { pattern }\end{array}$ & $\begin{array}{l}\text { Additional } \\
\text { uses }\end{array}$ \\
\hline $\begin{array}{l}\text { Polycarpon prostratum (Forssk.) } \\
\text { Asch. \& Schweinf. [Caryophyllaceae]; } \\
\text { Ajita \& AP Das 261 }\end{array}$ & - & Herb & $\begin{array}{l}\text { Whole plants are } \\
\text { eaten as } \\
\text { vegetable. }\end{array}$ & \\
\hline $\begin{array}{l}\text { Polygonum plebeium } \text { R.Br. } \\
\text { [Polygonaceae]; Ajita \& AP Das 119 }\end{array}$ & - & Herb & $\begin{array}{l}\text { Leafy-branches } \\
\text { cooked as } \\
\text { vegetable }\end{array}$ & \\
\hline $\begin{array}{l}\text { Portulaca oleracea } \text { L. [Portulacaceae]; } \\
\text { Ajita \& AP Das 456 }\end{array}$ & Hangshmoi & Herb & $\begin{array}{l}\text { Whole plants } \\
\text { cooked as } \\
\text { vegetable }\end{array}$ & \\
\hline $\begin{array}{l}\text { Solanum americanum Mill. } \\
\text { [Solanaceae]; Ajita \& AP Das 205 }\end{array}$ & Parbotaptoi & $\begin{array}{l}\text { Small } \\
\text { shrub }\end{array}$ & Fried leaves eaten & Medicinal \\
\hline $\begin{array}{l}\text { Typhonium trilobatum } \text { (L.) Schott } \\
\text { [Araceae]; Ajita \& AP Das 157 }\end{array}$ & Lheng & Herb & $\begin{array}{l}\text { Young leaves } \\
\text { pounded with } \\
\text { green chilli and } \\
\text { salt to consume as } \\
\text { pickle }\end{array}$ & \\
\hline
\end{tabular}

\section{LITERATURE CITED}

Adhikary, P. and Barman, D. 2014. The indigenous medicinal knowledge of the Rabha tribe: A case study in the Chirang district of Assam, North-East India. Life Sci. Leafl. 52: $94-97$.

Basumatary, P. 2010. The Rabha tribe of North-East India, Bengal and Bangladesh, Mittal publications, New Delhi, India.

Campbell, J.R. 2001. Participatory rural appraisal as qualitative research: distinguishing methodological issues from participatory claims. Human Organization. 60(4): 380 389.

Chowdhury, M. 2012. Chokoth a traditional alcoholic drink of Rabha tribe in india. Life Sci. Leafl. 4:54 - 59.

Das, C. and Teron, R. 2014. Ethnobotanical notes of the Rabha community in Mataikhar reserve forest of Kamrup district, Assam, India. Res. J. Recent Sci. 3(6): 26 - 33.

Egiye Bangla. 2018. District profiles. The official portal of the Government of West Bengal. Retrieved December 19, 2018. https://www.wb.gov.in/portal/web/guest/ district.

FAOUN (Food and Agriculture Organization of the United Nations). 2018. Food Security and Nutrition in the Age of Climate Change. Proceedings of the International Symposium organized by the Government of Québec in collaboration with FAO. Rome.

Mukherjee, N. 1993. Participatory rural appraisal: methodology and applications. Studies in rural participation 1. Concept Pub. Co., New Delhi. 
Nagoya Protocol on Access to Genetic Resources and the Fair and Equitable Sharing of Benefits arising from their Utilization to the Convention on Biological Diversity. 2011. Text and Annex Secretariat of the Convention on Biological Diversity, Montreal. Convention on Biological Diversity United Nations

Narzary, Y.; Brahma, J.; Brahma, C. \& Das, S. 2016. A study on indigenous fermented foods and beverages of Kokrajhar, Assam, India. J. Ethnic Foods. 3: 284 - 291.

Roy, M. 2016. A Historical Outline of the Rabhas of North Bengal. Imp. J. Interdis. Res. (IJIR) 2(3): $650-659$.

World Economic Situation and Prospects. 2018. United Nations Publication, New York.

Yongabi, K. \& DeLuca, L. 2015. Potentials of indigenous knowledge and ethnobiotechnology in sustainable agriculture in sub Saharan Africa. In: Sanjeev Kumar (ed.), Recent advances in ethnobotany. Deep Publications, New Delhi. pp. $41-56$. 